\title{
An Energy-Efficient Hierarchical Routing for Wireless Sensor Networks
}

\author{
Nishi Sharma \\ Rajasthan Technical University \\ Kota, India
}

\begin{abstract}
The popularity of Wireless Sensor Networks (WSN) has increased tremendously in recent time due to growth in MicroElectro-Mechanical Systems (MEMS) \& wireless communication technology. The main purpose of such networks is to collect information from the environments and deliver the same to the applications to determine characteristics of the environment or detect an event. As sensor nodes depend on batteries, they have limited amount of energy. One of the major issues in wireless sensor networks is to develop an energy-efficient routing protocol which has a significant impact on the overall lifetime of the sensor network. Many energy efficient routing protocols have been proposed to solve this problem and increase the lifetime of the network. This paper proposes a clustering technique which is based on LEACH protocol. In this, clusters are formed based on their geographical location \& cluster heads are selected on the basis of highest residual energy within the cluster as well as minimum distance to the base station from the cluster heads. The network lifetime for various levels of hierarchical routing techniques is compared. There are two outcomes from the implemented protocol. These are prolonged network lifetime \& increased mean residual energy with the increase in number of clusters in the wireless sensor network.
\end{abstract}

Keywords: Wireless Sensor Networks, Energy efficiency, Hierarchical routing, Improvement on LEACH protocol, Network lifetime.

\section{INTRODUCTION}

The emerging field of wireless sensor networks combines sensing, computation, and communication tasks into a single tiny device. A wireless sensor network can be generally described as a network of nodes that cooperatively sense and may control the environment enabling interaction between persons or computers and the surrounding environment. It is composed of a large number of sensor nodes that are densely deployed either inside the event area or very close to it. The position of sensor nodes needs not to be fixed. This allows random deployment of sensor networks in inaccessible terrains or disaster relief operations. Sensor nodes are fitted with an onboard processor. Instead of sending the raw data to the other nodes, they use their processing abilities to locally carry out simple computations and transmit only the required and partially processed data [1]. A greater number of sensors allows for sensing over larger geographical regions with greater accuracy. Sensor nodes coordinate among themselves to produce high-quality information about the physical environment. Each of these scattered sensor nodes has the capability to collect and route data either to other sensors or back to an external base station(s). A base-station should be capable of connecting the sensor network to an existing communications infrastructure or to the Internet where a user can have access to the reported data [2]. The sensor nodes depend on batteries as their energy sources which means they are constrained in terms of energy. In order to achieve longer network lifetime, energy efficient routing protocols are required.

The Routing protocols are broadly divided as Flat, Hierarchical \& Location based routing [2]. The hierarchical or clustering technique is considered as a good method to minimize energy consumption in WSN. It is mainly used by the sensor nodes, in which sensors send information to only the cluster heads and then the cluster head transmits the aggregated information to the base stations or the sink. In this, the nodes are often grouped together into disjoint and mostly non-overlapping clusters to minimize communication latency and improve energy efficiency. Leader of every cluster is often referred to as the cluster-head. A cluster-head may be elected by the sensors in a cluster or pre-assigned by the network designer. A cluster-head may also be just one of the sensors or a node that is richer in resources. Cluster-heads may form a second tier network or may just ship the data to the base-station. The advantage of this scheme is that it reduces energy usage of each node and communication cost. In this technique [3], nodes perform different tasks in WSNs and typically are organized into lots of clusters according to specific requirements or metrics. In general, nodes having higher energy act as cluster head and perform the task of data processing and information transmission, while nodes with low energy act as member nodes and perform the task of sensing the information. The typical clustering routings protocols in WSNs include LEACH, PEGASIS, TEEN, APTEEN and HEED etc for wireless sensor network. The organization of the paper is as follows. Section I gives introduction in brief, section II explains about LEACH protocol, section III describes the Sensor Network Model. Section IV gives proposed protocol. Section V gives Simulation results of non-hierarchical \& hierarchical routing protocols using MATLAB. Section VII concludes the paper.

\section{DESCRIPTION OF LEACH PROTOCOL}

Hierarchical based routing protocols provide data aggregation, scalability, less load, less energy consumption and more robustness, more fault tolerance as compare to flat based routing protocols [3]. Heinzelman et al. proposed Low-Energy Adaptive Clustering Hierarchy (LEACH) [4] for efficient routing of data in wireless sensor networks. LEACH is a selforganizing, adaptive clustering protocol that uses randomization to distribute the energy load evenly among the sensors in the network. In LEACH, the nodes are organized themselves into clusters, with one node behaving as the cluster-head. If the cluster heads were chosen a priori and 
fixed throughout the system lifetime, they would die quickly, ending the lifetime of all nodes belonging to those clusters. Thus LEACH uses randomized rotation of the high-energy cluster-head position such that it rotates among the various sensors so that the battery of a single sensor would not drain quickly. LEACH also performs data fusion to compress the amount of data being sent from the clusters to the base station, thereby reducing energy dissipation and enhancing system lifetime. Sensor nodes elect themselves as cluster-heads at any given time with a certain probability. These cluster head nodes advertise their status to the other nodes in the network. Each sensor node determines the cluster to which it wants to belong by choosing the cluster-head that requires the minimum communication energy for transmission. Once clusters are formed, each cluster-head broadcasts a TDMA schedule and assign each node a time slot in which it can transmit the sensed data. The non-cluster head nodes sense the data and send it to their cluster-head according to the TDMA schedule. Once the cluster-head collects all the data from the nodes in its cluster, they aggregate the data and then transmit the compressed data to the base station. Since the base station is far away in the scenario we are examining, this is a high energy transmission. The main drawbacks of LEACH protocol are:

- Cluster heads are selected randomly in LEACH; it is possible that nodes with less energy would be chosen, which could lead to these nodes die too fast.

- In addition, because in LEACH protocol cluster heads communicate with base stations in single-hop manner, it is energy consuming and its expandability is limited so that it could not adapt to large network.

Both of these shortcomings will be overcome by the proposed protocol.

\section{SENSOR NETWORK MODEL}

There has been a significant amount of research in the area of low-energy radios. In this work, we assume a simple model where the transmitter dissipates energy to run the radio electronics and the power amplifier and the receiver dissipates energy to run the radio electronics $[4,5]$ as shown in Figure 1. The power attenuation is dependent on the distance between the transmitter and receiver. The propagation loss will be inversely proportional to $\mathrm{d}^{2}$ for relatively short distances, whereas it will be inversely proportional to $\mathrm{d}^{4}$ for longer distances. Thus, to transmit a k-bit message to distance d, the radio expends:

$$
\begin{aligned}
& E_{T X}(k, d)=k * E_{\text {elec }}+E_{\text {amp }}(k, d) \\
& E_{T X}(k, d)= \begin{cases}k^{*} E_{\text {elec }}+k * \varepsilon_{f s} * d^{2} & \text { if } d<d_{0} \\
k * E_{\text {elec }}+k * \varepsilon_{m p} * d^{4} & \text { if } d \geq d_{0}\end{cases}
\end{aligned}
$$

and to receive this message, the radio expends:

$$
E_{R X}(k)=E_{\text {elec }} * k
$$

Here

$\mathrm{E}_{\mathrm{TX}}(\mathrm{k}, \mathrm{d})$ - energy dissipated per bit at transmitter

$\mathrm{E}_{\mathrm{RX}}(\mathrm{k}, \mathrm{d})$ - energy dissipated per bit at receiver
$\mathrm{E}_{\text {elec }}$ energy required while transmitting or receiving one bit of data

\section{$\mathrm{E}_{\mathrm{amp}}$ - amplifier coefficient}

d - distance between a sensor node and its respective cluster head or between a cluster head to another cluster head nearer to the base station or between cluster head and base station.

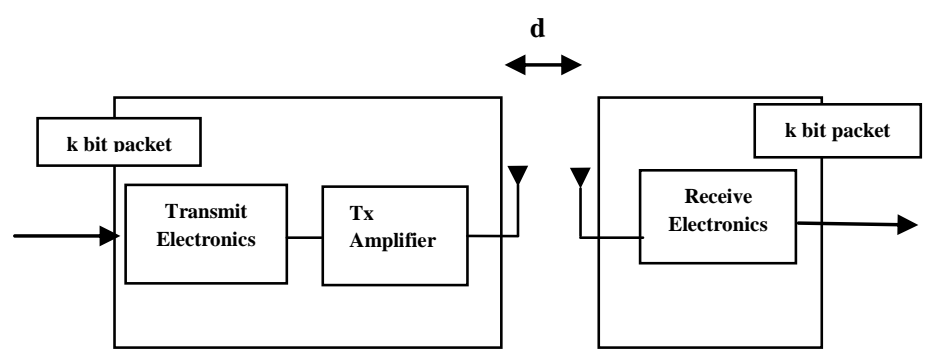

Figure 1: First order radio model

\section{PROPOSED ROUTING ALGORITHM}

This paper introduces an Improvement to LEACH protocol which increases the lifetime of the sensor network as well as mean residual energy of the network. In the proposed protocol, clusters are formed geographically. The sensing area is divided into the equal parts, nodes belongs to the same part forms the cluster. In case of non-hierarchical cluster formation, entire sensor area space will be used. But in other cases such as two, three $\&$ four cluster formation, sensor area space will be divided into equal areas. The two, three \& four clusters formation are otherwise known as first level, second $\&$ third level hierarchy respectively. In case of LEACH this equal area segregation is not used.

Once the clusters are formed, cluster-head selection phase starts. In this work, we make use of first order radio model to make some energy calculations. We first calculate the residual energy of each node of each cluster. Then the energy of the nodes within a cluster is compared to each other. In order to do efficient communication, the node having maximum energy in the clusters is selected as cluster-heads. Since the cluster head performs data collection from various sensor nodes within the cluster, data aggregation \& data transmission, they lose their energy very quickly. Due to draining activities being constraint on a cluster head; the cluster-head is rotated among the sensor nodes of each cluster at every transmission round. After each transmission the residual energy of the nodes is recalculated and then again comparing the nodes energy and selecting the node with maximum energy as the cluster-head. By rotating the cluster heads on the basis of residual energy, we can equally divide the burden of transmission \& thereby increase the network lifetime.

In this approach we also use minimum distance concept during transmission. We calculate the distance between nodes to cluster heads and cluster heads to other promising cluster heads or to the base station. Then the minimum distance path is selected for the transmission so that less energy is wasted in transmitting data. Once the cluster head with shortest path is selected, they aggregate the data to be transmitted and then transmit it using shortest path.

The proposed hierarchical routing algorithm can be summarized using following steps (Figure 2): 
- Cluster formation is done by dividing the area into equal parts.

- Cluster heads are selected from each cluster on the basis of residual energy as well as the shortest distance to the base station.

- Data aggregation phase which involves the gathering of collected data by the cluster head from the sensor nodes within its cluster.

- Data transmission phase in which data is transferred from the cluster heads to other $\mathrm{CHs}$ or to the base stations.

The Cluster Head selection process used in proposed technique can be described as follows:-

- The initial energy $E_{\text {in }}(n)$ of each node is measured.

- Also, the distance d(n) from each node to the base station or to the next higher level cluster head is calculated.

- Then we compare the measured distances \& select the minimum distance for the transmission in that round.

- Estimation of the energy required by each node for transmission within the cluster (not to BS or to higher level $\mathrm{CH}$ ) is carried out using the formula: $\left(E_{\text {amp }} * \mathrm{k}^{2}\right)$. The residual energy of each node is given by

$$
E_{\text {in }}(n)=E_{\text {in }}(n)-\left(E_{\text {elec }} * k+E_{\text {amp }} * k * d^{2}\right)
$$

- The cluster head is then selected on the basis of maximum residual energy in that round. After the $\mathrm{CH}$ selection is done, the next cluster head selection will take place after the current round is completed.

This cluster head selection process is shown in Figure 3.

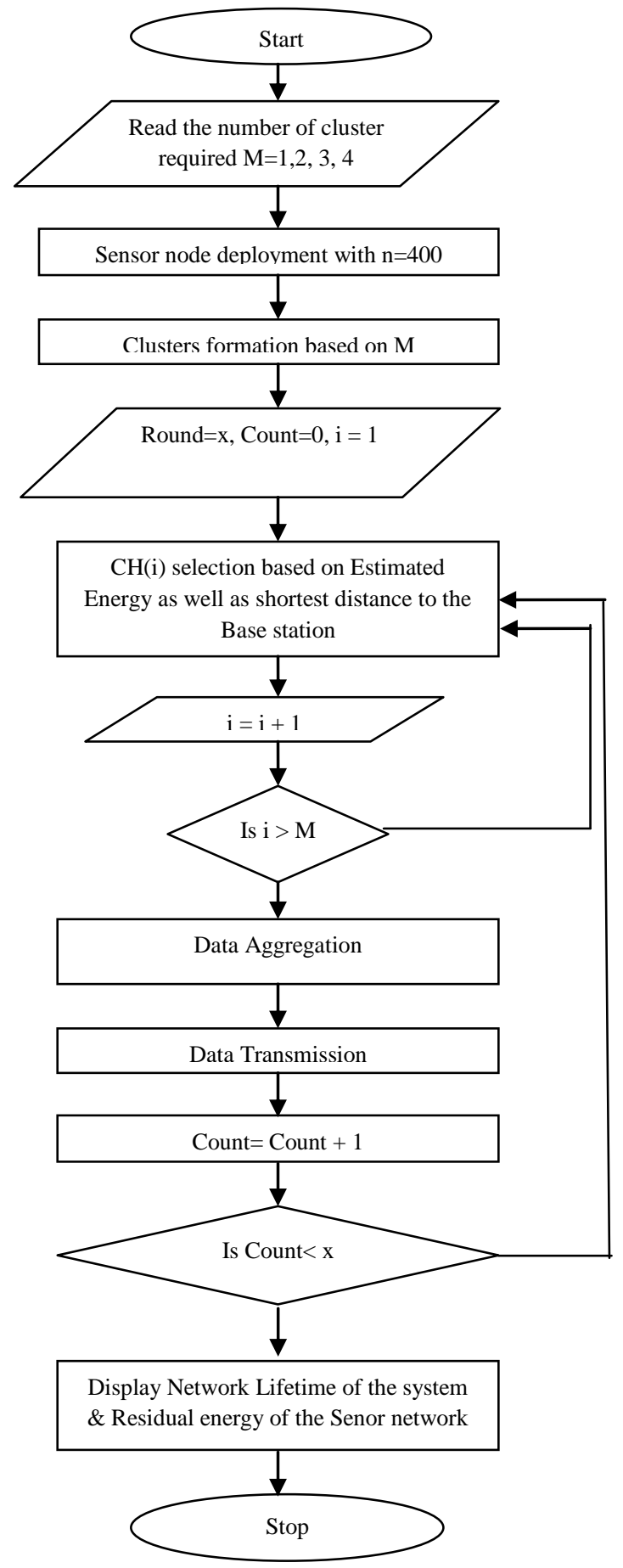

Figure 2: Flow chart of Proposed Routing Technique 


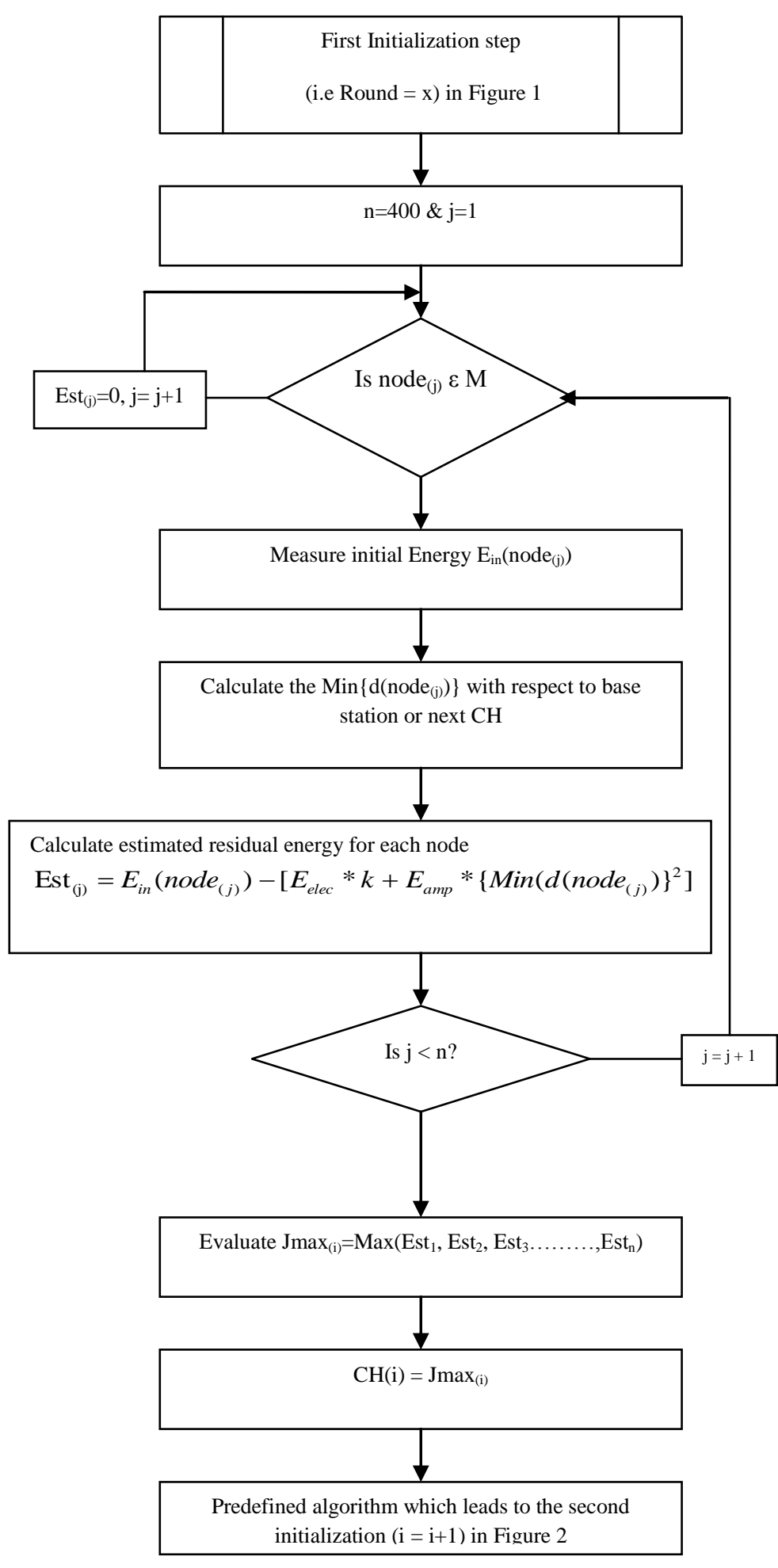

Figure 3: Flowchart of Cluster head selection process

\section{SIMULATION \& PERFORMANCE EVALUATION}

In this section we present the results of experiments carried out for evaluating the performance of the proposed hierarchical based routing for one, two \& three clusters, four. Simulation experiments are carried out in MATLAB. We also show how this protocol increases network lifetime \& residual energy as the number of clusters is increased.

Throughout the simulation, 300 sensor nodes are deployed in $300 \times 300$ regions. The size of sensor data packet is $100 \mathrm{~K}$ bytes. We use first order radio model for data reception, aggregation \& transmission by the nodes in the sensor networks. Nodes' are initialized with 200J energy. Then we compare the results with the network of nodes having initial energy 300J. Results show that network lifetime increases when we increase the initial energy of the nodes. Simulation parameters are shown in table 1 .

Table 1: Simulation parameters

\begin{tabular}{|c|c|}
\hline Parameter & Value \\
\hline Number of Nodes & 300 \\
\hline Network size $\left(\mathrm{m}^{2}\right)$ & $300 \times 300$ \\
\hline Base station location & $(0,0)$ \\
\hline Nodes Initial Energy & $200 \mathrm{~J} / 300 \mathrm{~J}$ \\
\hline Packet size (Kbytes) & 100 \\
\hline $\mathrm{E}_{\text {elec }}(\mathrm{nJ} / \mathrm{bit})$ & 50 \\
\hline $\mathrm{E}_{\mathrm{amp}}(\mathrm{pJ} / \mathrm{bit})$ & 100 \\
\hline
\end{tabular}

For simplicity, we assume the following:

- All nodes are homogeneous in nature;

- All nodes begins with the same initial energy;

- Clusters and nodes are static. This means nodes' location is fixed throughout the operation;

- Normal nodes transmit the data directly to their respective cluster heads within a particular cluster;

Cluster heads use multi-hop routing to relay data to the base station. They can transmit data either to base station or to next promising cluster heads.

\subsection{Node Deployment}

The sensor nodes in the network are formed into clusters of different sizes of one, two, three \& four. One indicates a Nonhierarchical formation of cluster whereas two, three \& four indicate First, Second \& Third level hierarchy. Figure 4 indicates the non-hierarchical structure of our routing technique. Likewise, Figure 5 and 6 shows the simulation result of two \& three cluster formation using proposed hierarchical technique. We also show the simulation result of four clusters formation scenario which indicates Third level hierarchical technique. In this case formation of clusters is done in two ways (Case I \& II) shown in Figure $7 \& 8$. 


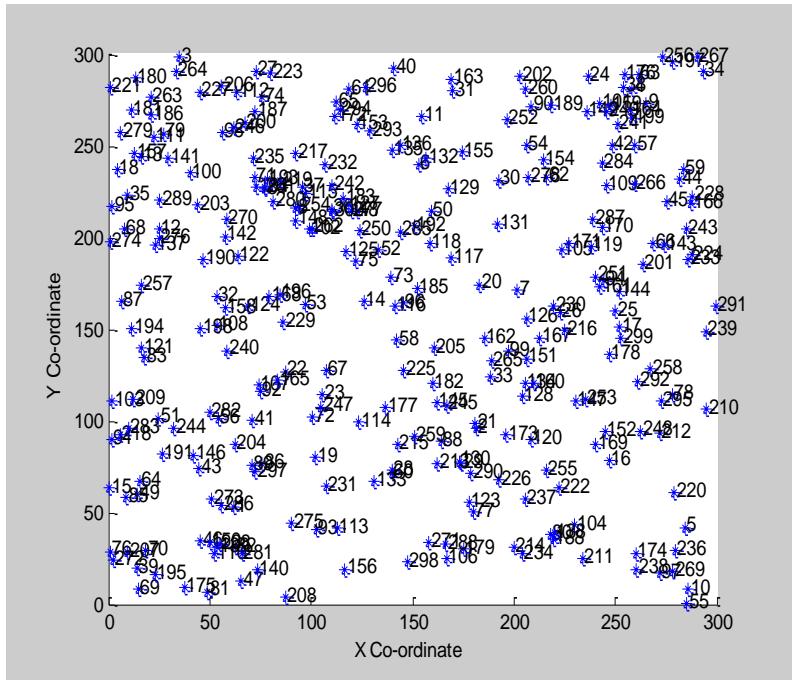

Figure 4: Non hierarchical cluster formation

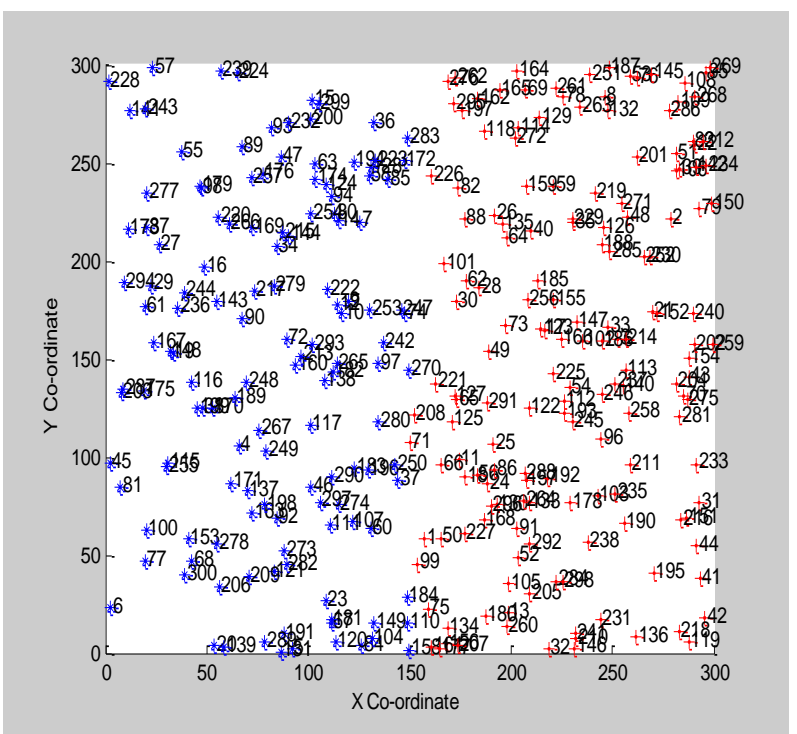

Figure 5: First level hierarchical cluster formation

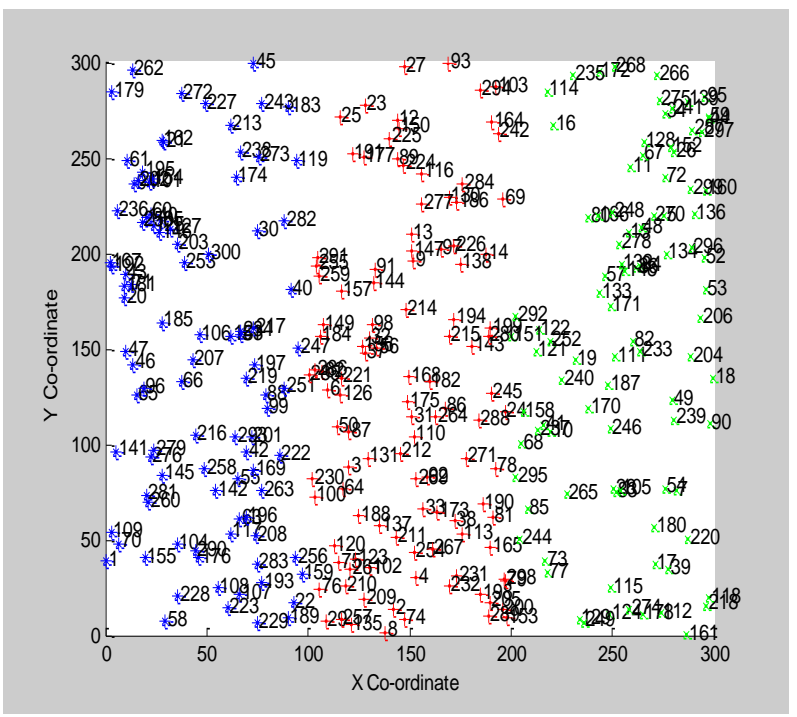

Figure 6: Second level hierarchical cluster formation

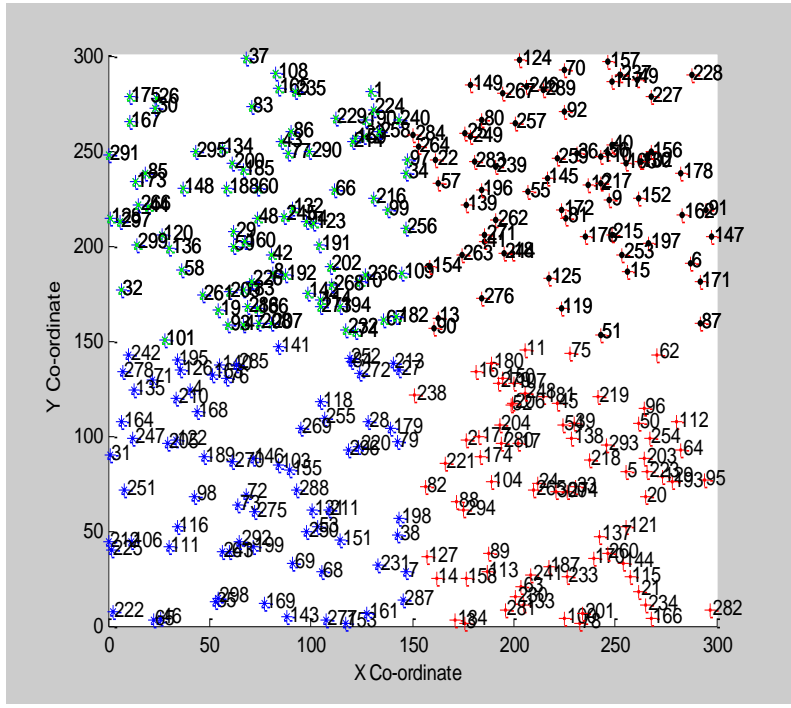

Figure 7: Third level hierarchical cluster formation

(Case I)

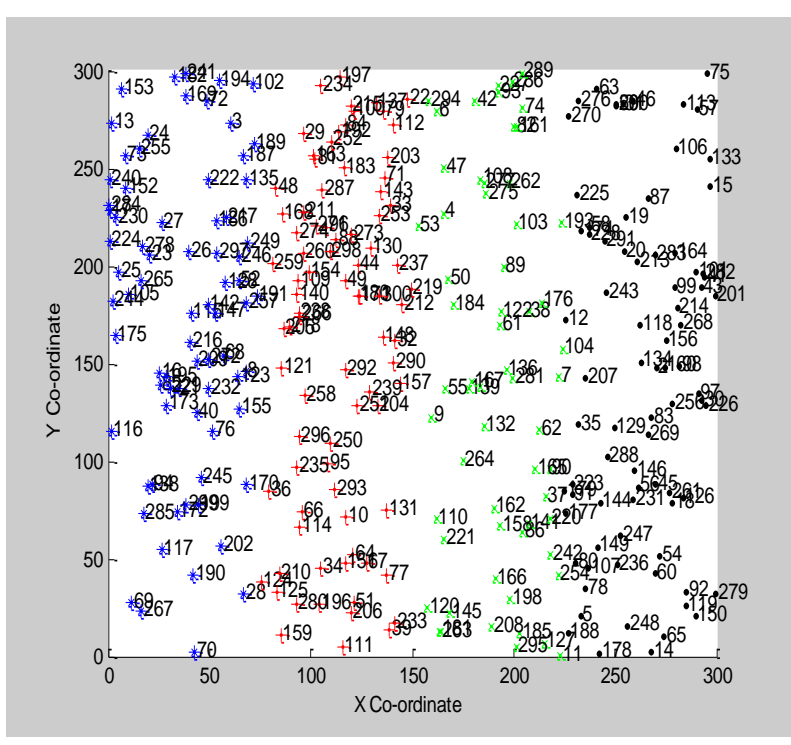

Figure 8: Third level hierarchical cluster formation

\section{(Case II)}

\subsection{Network Lifetime}

One of the most important factors for evaluating the sensor network is network lifetime. The lifetime of the network depends on the lifetime of each sensor node that is a part of that network. Recharging and replacing the nodes' batteries is impractical in many environments. In our experiment we define the network lifetime as the time until all the nodes are dead. The network lifetime for non hierarchical, First level, Second level, Third level hierarchical routing is shown in Figure $9,10,11,12, \& 13$. We observed that the nodes begin to die more quickly in the non-hierarchical routing technique since all nodes in the network send captured data via one randomly selected cluster head per round to the base station. 
We observed that the proposed technique offers improvement in network lifetime as we increase the number of clusters in the network. Non-hierarchical technique network completely stopped functioning at an earlier simulation rounds compared to our proposed technique. We observed that Case II of Third level hierarchy gives better results than Case I. Table 2 displays number of alive nodes with the increase in number of clusters when the initial energy of all the nodes are 200 Joules. We further increase the initial energy of all the nodes to 300 Joules. Results are shown in Table 3 . We observed that when we increases the initial energy of all the nodes, network lifetime will be increased.

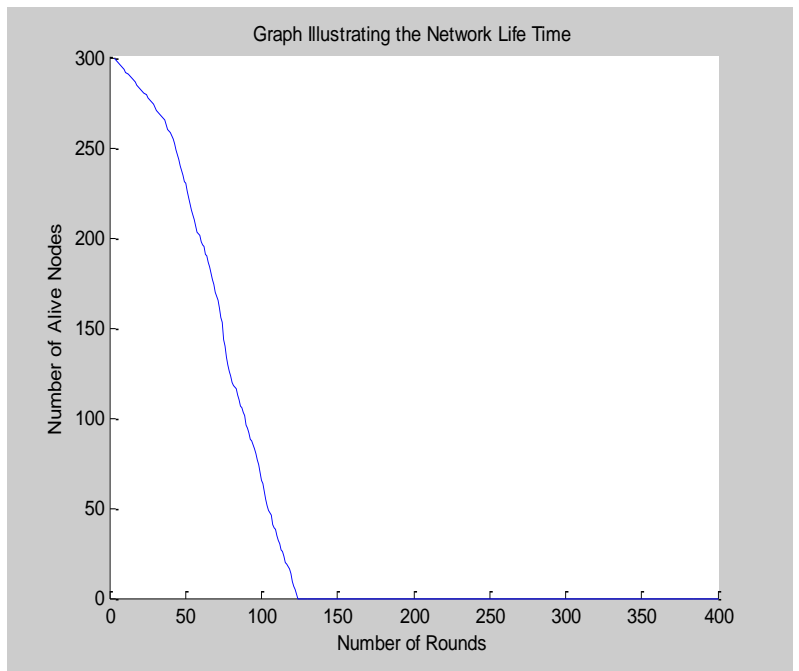

Figure 9: Network lifetime graph (Non-hierarchical Routing protocol)

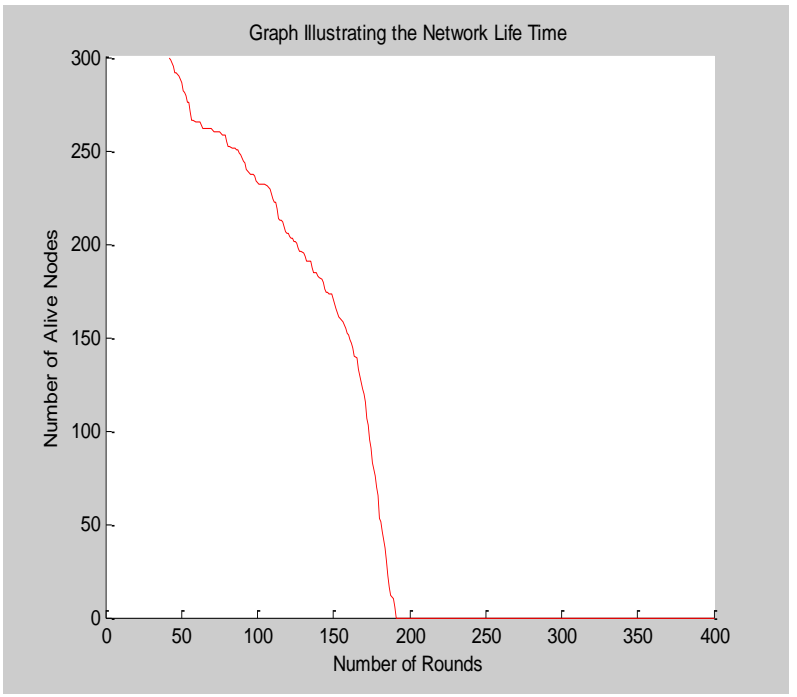

Figure 10: Network lifetime graph (First-level hierarchical Routing protocol)

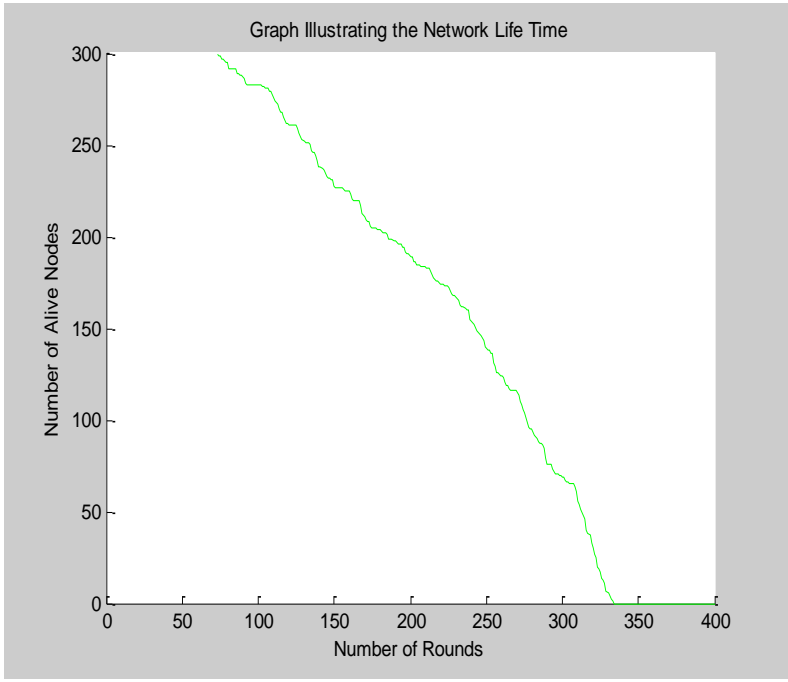

Figure 11: Network lifetime graph (Second-level hierarchical Routing protocol)

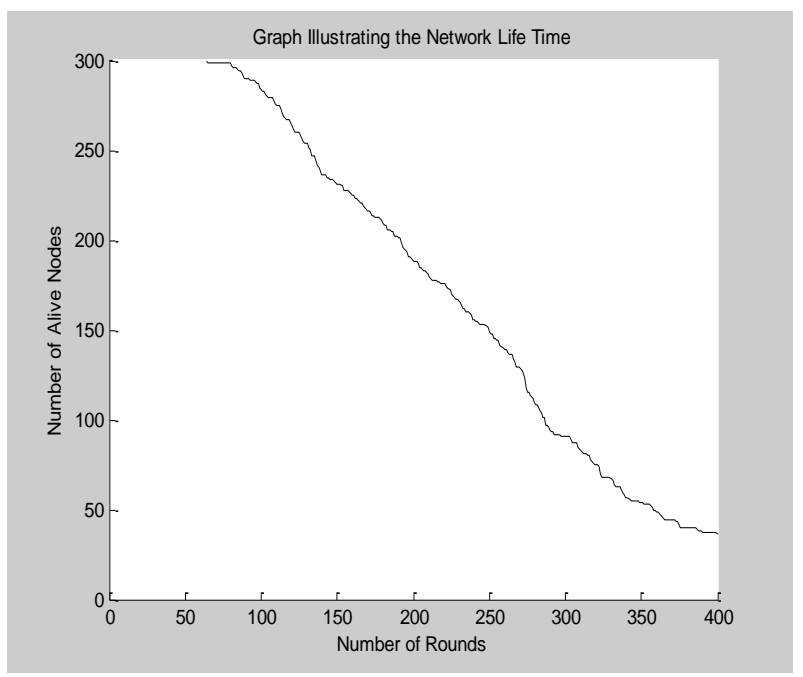

Figure 12: Network lifetime graph (Third-level hierarchical Routing protocol Case I)

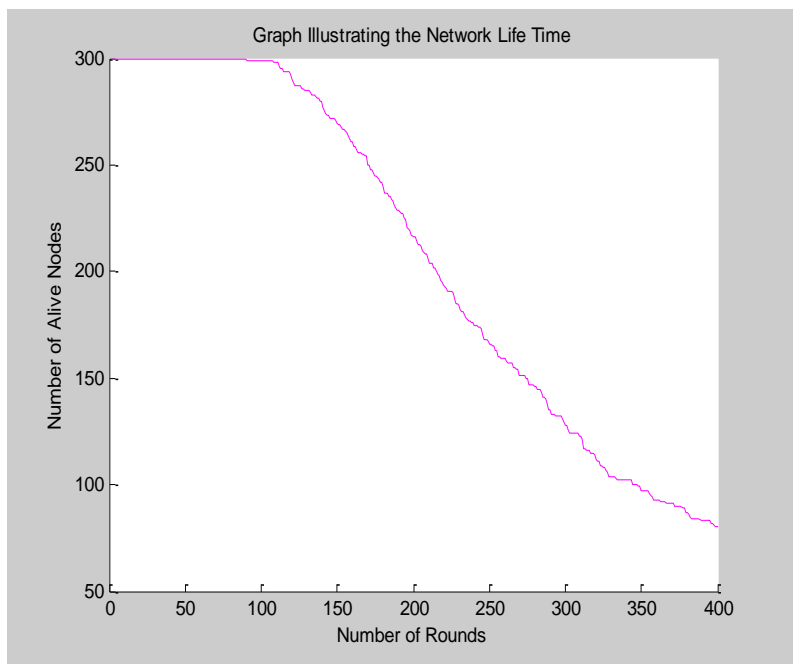

Figure 13: Network lifetime graph (Third-level hierarchical Routing protocol Case II) 
Table II: Network Lifetime Comparison

(Initial Energy=200Joules)

\begin{tabular}{|c|l|l|l|l|l|}
\hline & $\begin{array}{c}\text { First } \\
\text { Node } \\
\text { Dies }\end{array}$ & $\begin{array}{c}\text { Half } \\
\text { Nodes } \\
\text { Dies }\end{array}$ & $\begin{array}{c}\text { Last } \\
\text { Nodes } \\
\text { Dies }\end{array}$ & $\begin{array}{c}\text { Network } \\
\text { Lifetime }\end{array}$ & $\begin{array}{c}\text { Nodes } \\
\text { alive } \\
\text { till } \\
\text { last } \\
\text { round }\end{array}$ \\
\hline $\begin{array}{c}\text { Non- } \\
\text { hierarchy }\end{array}$ & 4 & 74 & 124 & 120 & 0 \\
\hline $\begin{array}{c}\text { First level } \\
\text { Hierarchy }\end{array}$ & 43 & 161 & 191 & 148 & 0 \\
\hline $\begin{array}{c}\text { Second level } \\
\text { Hierarchy }\end{array}$ & 74 & 243 & 334 & 260 & 0 \\
\hline $\begin{array}{c}\text { Third level } \\
\text { Hierarchy } \\
\text { (Case I) }\end{array}$ & 65 & 249 & - & - & 36 \\
\hline $\begin{array}{c}\text { Third-level } \\
\text { Hierarchy } \\
\text { (Case II) }\end{array}$ & 91 & 271 & - & - & \\
\hline
\end{tabular}

\subsection{Mean Residual Energy}

We evaluate the residual energy of each node for particular rounds of simulation. Simulation results in Figure 14, $15 \& 16$ show residual energy of nodes in non-hierarchical, first level \& second level hierarchical routing technique respectively. Results show that the mean residual energy value of nodes in the proposed hierarchical technique is higher than non hierarchical technique. This implies improved network performance since the nodes has more energy. We also evaluate the mean residual energy of nodes for third level hierarchy (four cluster formation). Simulation results in Figure $17 \& 18$ shows increased residual energy as compare non hierarchical, first \& second level hierarchy. Case II performs better than case I in terms of residual energy. The mean value of the residual energy increases as the number of cluster increases.
Table III: Network Lifetime Comparison

(Initial Energy=300Joules)

\begin{tabular}{|c|l|l|l|l|l|}
\hline & $\begin{array}{c}\text { First } \\
\text { Node } \\
\text { Dies }\end{array}$ & $\begin{array}{c}\text { Half } \\
\text { Nodes } \\
\text { Dies }\end{array}$ & $\begin{array}{c}\text { Last } \\
\text { Nodes } \\
\text { Dies }\end{array}$ & $\begin{array}{l}\text { Network } \\
\text { Lifetime }\end{array}$ & $\begin{array}{c}\text { Nodes } \\
\text { alive } \\
\text { till last } \\
\text { round }\end{array}$ \\
\hline $\begin{array}{c}\text { Non- } \\
\text { hierarchy }\end{array}$ & 7 & 127 & 197 & 190 & 0 \\
\hline $\begin{array}{c}\text { First level } \\
\text { Hierarchy }\end{array}$ & 187 & 375 & - & - & 61 \\
\hline $\begin{array}{c}\text { Second } \\
\text { level } \\
\text { Hierarchy }\end{array}$ & 189 & - & - & - & 188 \\
\hline $\begin{array}{c}\text { Third } \\
\text { level } \\
\text { Hierarchy } \\
\text { (Case I) }\end{array}$ & 127 & - & - & - & 155 \\
\hline $\begin{array}{c}\text { Third- } \\
\text { level } \\
\text { Hierarchy } \\
\text { (Case II) }\end{array}$ & 236 & - & - & - & \\
\hline
\end{tabular}

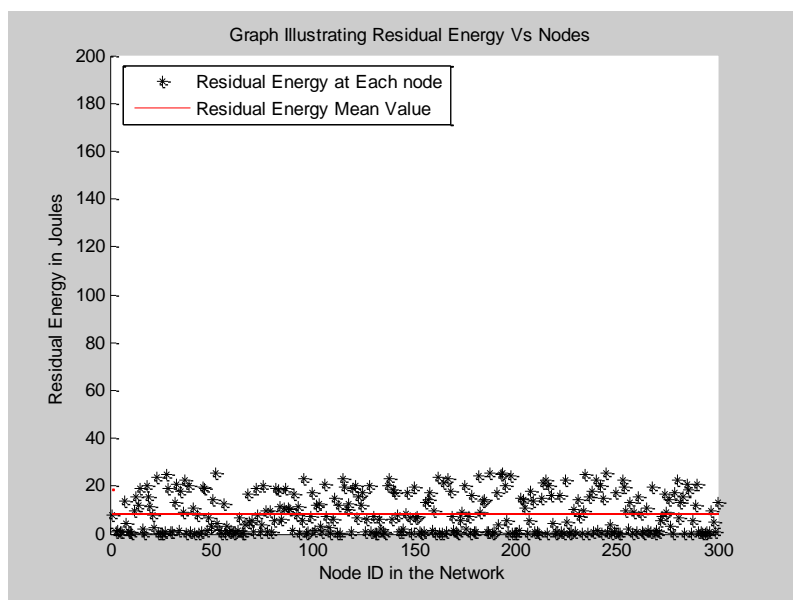

Figure 14: Nodes energy residue in non hierarchical technique after 400 rounds of simulations. 


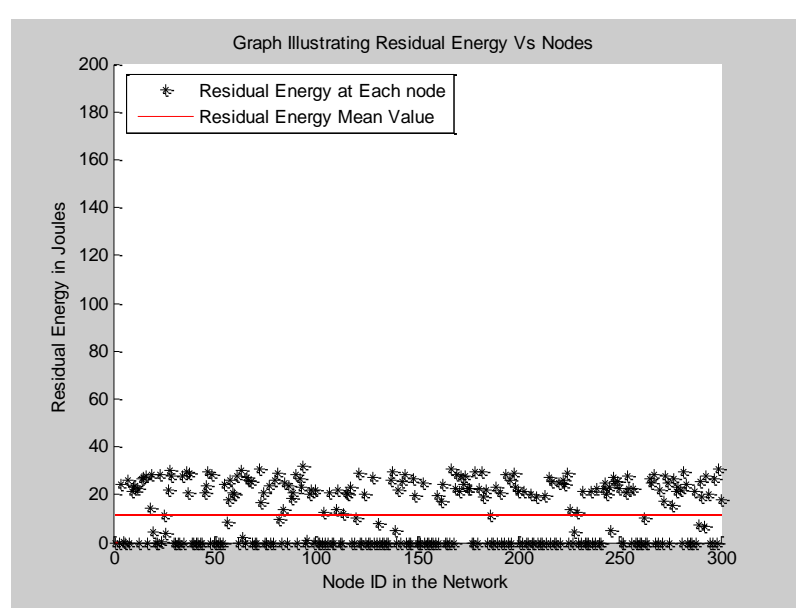

Figure 15: Nodes residual energy in First-level hierarchical technique after 400 rounds of simulations.

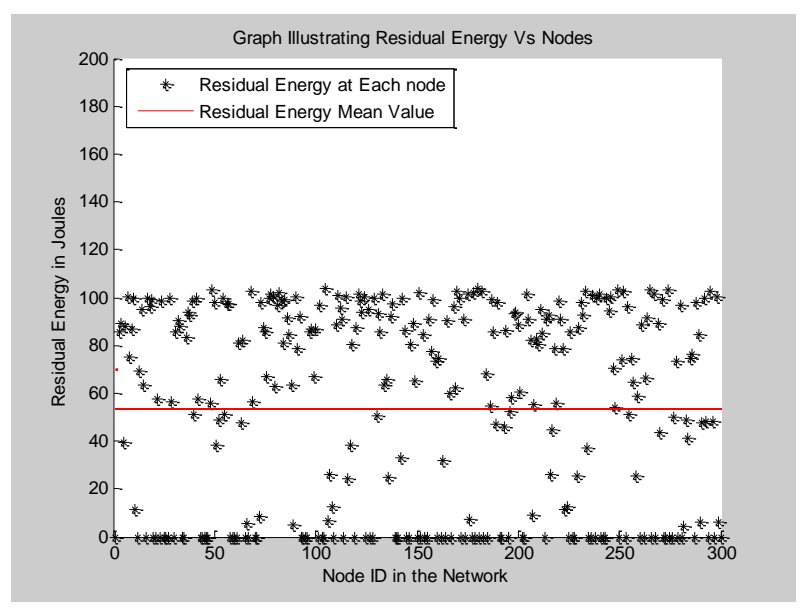

Figure 16: Nodes residual energy in Second-level hierarchical technique after 400 rounds of simulations.

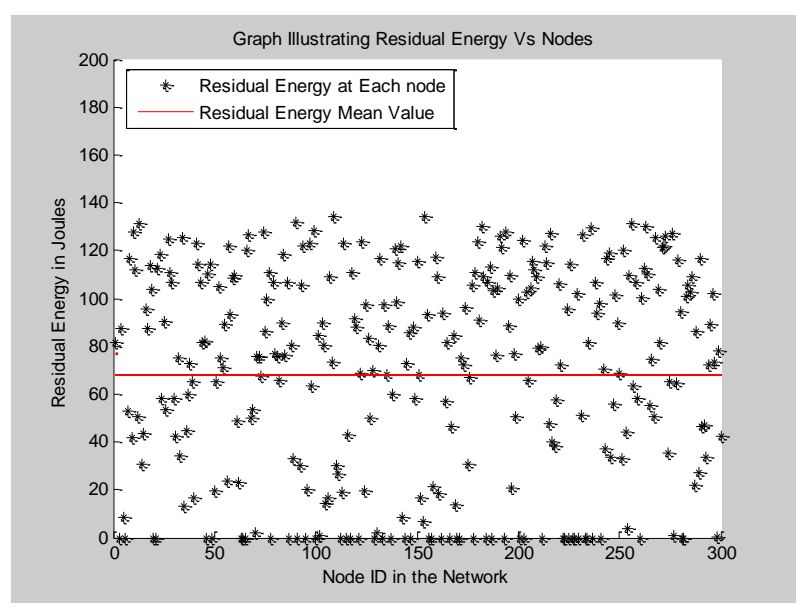

Figure 17: Nodes residual energy in Third-level hierarchical technique after 400 rounds of simulations (Case I).

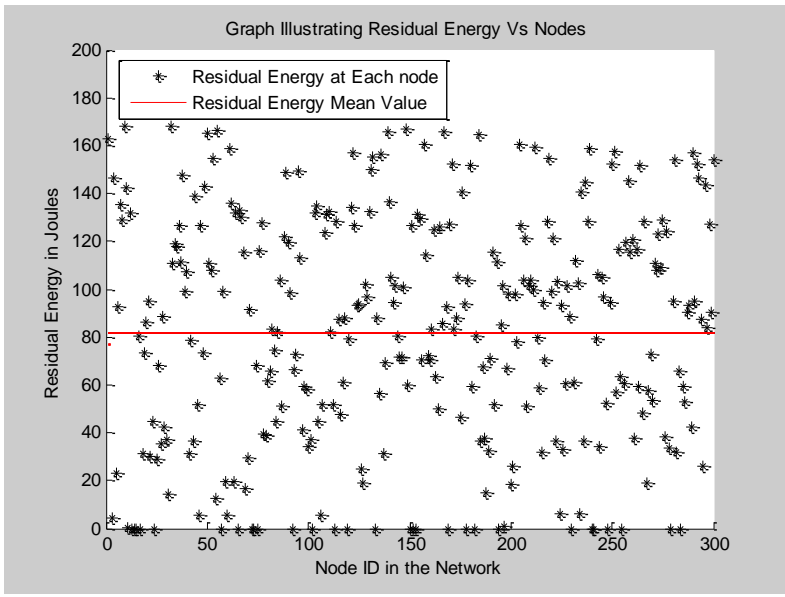

Figure 18: Nodes residual energy in Third-level hierarchical technique after 400 rounds of simulations (Case II)

Simulation results are shown in Table 4 when the initial energy of the nodes are 200 Joules. We also observed that the residual energy of the nodes \& network is also increased when we increases the initial energy of the nodes to 300 Joules. These results are shown in Table 5.

Table IV: Comparison of Mean \& Variance of Residual Energy (Initial Energy=200 Joules)

\begin{tabular}{|l|c|c|}
\hline & $\begin{array}{c}\text { Mean residual } \\
\text { energy (Joules) }\end{array}$ & $\begin{array}{c}\text { Variance residual } \\
\text { energy (Joules) }\end{array}$ \\
\hline Non-hierarchical & 8.33 & 8.05 \\
\hline $\begin{array}{l}\text { First-level } \\
\text { hierarchy }\end{array}$ & 11.68 & 12.00 \\
\hline $\begin{array}{l}\text { Second-level } \\
\text { hierarchy }\end{array}$ & 53.65 & 41.62 \\
\hline $\begin{array}{l}\text { Third-level } \\
\text { hierarchy } \\
\text { (Case I) }\end{array}$ & 67.95 & 44.24 \\
\hline $\begin{array}{l}\text { Third-level } \\
\text { hierarchy } \\
\text { (Case II) }\end{array}$ & 81.77 & 49.22 \\
\hline
\end{tabular}

Table V: Comparison of Mean \& Variance of residual energy (Initial Energy=200 Joules)

\begin{tabular}{|l|c|c|}
\hline & $\begin{array}{c}\text { Mean residual } \\
\text { energy (Joules) }\end{array}$ & $\begin{array}{c}\text { Variance residual } \\
\text { energy (Joules) }\end{array}$ \\
\hline Non-hierarchical & 11.31 & 8.65 \\
\hline $\begin{array}{l}\text { First-level } \\
\text { hierarchy }\end{array}$ & 85.61 & 40.64 \\
\hline $\begin{array}{l}\text { Second-level } \\
\text { hierarchy }\end{array}$ & 132.90 & 66.10 \\
\hline $\begin{array}{l}\text { Third-level } \\
\text { hierarchy } \\
\text { (Case I) }\end{array}$ & 101.31 & 65.11 \\
\hline $\begin{array}{l}\text { Third-level } \\
\text { hierarchy } \\
\text { (Case II) }\end{array}$ & 178.35 & 62.47 \\
\hline
\end{tabular}




\section{CONCLUSION}

Simulation results show that the proposed hierarchical routing technique gives better results when compared to nonhierarchical technique. We observed that network lifetime \& residual energy of the network is increased when we increase the number of clusters. This means that network remains alive for a longer time so more transmission can be done when proposed technique is used. This also shows that when we increase the initial energy of each node, the network lifetime $\&$ residual energy will also be increased.

\section{REFERENCES}

[1] I. F. Akyildiz, W. Su, Y. Sankarasubramaniam, and E. Cayirci, "A survey on sensor networks," Communications Magazine, IEEE, vol. 40, pp. 102-114, 2002.

[2] Al-Karaki, J. N. and A. E. Kamal, "Routing Techniques in Wireless Sensor Networks: A Survey," IEEE Wireless Communications, 11(6), Dec., 2004.

[3] Xu-Xun Liu “ A survey on Clustering Routing Protocols in Wireless Sensor Networks", Sensors 2012.

[4] W.Heinzelman, A.Chandrakasanand and $H$. Balakrishnan, "Energy-efficient communication protocol for wireless microsensor networks," in Proceedings of the 33rd Annual Hawaii International Conference on System Sciences, Maui, HI, 2000, pp. 3005-3014.

[5] W.B. Heinzelman, A.P. Chandrakasan, H. Balakrishnan, "Application specific protocol architecture for wireless microsensor networks," Wireless Communications, IEEE, vol. 1, pp. 660-670, october 2002. 